\title{
Reconstruction of a distribution from a finite number of its moments: a comparative study in the case of depolymerization process
}

\author{
Noureddine Lebaz ${ }^{\mathrm{a}, \mathrm{b}, \mathrm{c}, \mathrm{d}, *}$, Arnaud Cockx ${ }^{\mathrm{b}, \mathrm{c}, \mathrm{d}}$, Mathieu Spérandio ${ }^{\mathrm{b}, \mathrm{c}, \mathrm{d}}$, Jérôme Morchain ${ }^{\mathrm{b}, \mathrm{c}, \mathrm{d}}$ \\ ${ }^{a}$ Toulouse White Biotechnology (UMS INRA/INSA/CNRS), 3 rue Ariane, 31520 Ramonville Saint Agne, France \\ ${ }^{b}$ Université de Toulouse; INSA, UPS, INP; LISBP, 135 Avenue de Rangueil, F-31077, Toulouse, France \\ ${ }^{c}$ INRA, UMR792 Ingénierie des Systèmes Biologiques et des Procédés, F-31400, Toulouse, France \\ ${ }^{d}$ CNRS, UMR5504, F-31400, Toulouse, France
}

\begin{abstract}
The resolution of the Population Balance Equation (PBE) using moment-based methods offers a high computational efficiency however, information on the time evolution of the probability density function (PDF) is out of reach. For this, several PDF reconstruction methods using a finite number of moments are proposed in the literature. In this contribution, three different methods (i.e. Beta Kernel Density Function based method, Spline based technique and the Maximum Entropy based approach) are tested and compared to the analytical solution of a depolymerization process. The Maximum Entropy method gives the most accurate approximations using only a set of six moments. This method is combined with the Quadrature Method of Moments (QMOM) for a simultaneous reconstruction during the PBE resolution. A three nodes and a four nodes quadrature are tested. The results show that the quality of the reconstruction is highly dependent on the accuracy of the computed moments.
\end{abstract}

Keywords: Moment problem, population balance, reconstruction methods, maximum entropy

\section{Introduction}

The recovery of a probability density function (PDF) knowing only a finite number of its moments is known as the finite-moment problem in mathematical analysis and arises in different scientific applications (e.g. physics, chemical engineering, economics) (Gavriliadis \& Athanassoulis, 2012, John et al., 2007). This 5 problem is generally declined in three different problem categories for the mono-variate case (Abramov, 2007):

- The Hausdorff moment problem: the PDF is supported on the closed interval $[a, b]$

- The Stieltjes moment problem: the PDF is supported on $[0,+\infty)$

- The Hamburger moment problem: the PDF is supported on the real line $(-\infty,+\infty)$

In chemical engineering, especially for particulate/dispersed systems (e.g. crystallization, polymerization/depolymerization, liquid-liquid extraction, multiphase systems), population balance models (PBM) are

\footnotetext{
${ }^{*}$ Corresponding author. Address: INSA, LISBP, 135, avenue de Rangueil, F-31077 Toulouse, France. Tel.: +33 56 155 9798; Fax: +33561559760

Email address: lebaz@insa-toulouse.fr (Noureddine Lebaz)
} 
widely used for the description of the time evolution of the variable-based distribution (e.g. size, volume) undergoing elementary processes. Among these processes, one commonly finds breakage, aggregation/coalescence, nucleation, growth/dissolution ... etc (Ramkrishna \& Mahoney, 2002). The resolution of the population balance equation (PBE) is computationally intensive when using classical approaches (e.g. Monte Carlo methods (Lin et al., 2002), discretization methods (Kumar \& Ramkrishna, 1996)). This drawback is limiting when population balance modelling is coupled with Computational Fluid Dynamics (CFD) which is the case for multiphase systems. In order to overcome this issue, moment based methods (MOM: Standard Method of Moments (Hulburt \& Katz, 1964), QMOM: Quadrature Method of Moments (McGraw, 1997: Marchisio et al., 2003a), DQMOM: Direct Quadrature Method of Moments (Marchisio \& Fox, 2005)) offering by the fact a high computational efficiency. In QMOM the transported moments are calculated by reducing the PDF to an n-point distribution (sum of $n$ weighted Dirac delta functions), the corresponding weights and abscissas are computed using specific algorithms (John \& Thein, 2012). In DQMOM, the weights and the abscissas of the initial n-point distribution are directly tracked instead of the moments.

Even though the moment-based methods are computationally efficient, information regarding either the shape or pointwise values of the PDF are identically out of reach. However, this level of knowledge is important for different applications as in the case of evaporation process where one needs to accurately evaluate the PDF at zero size (Massot et al. 2010) or in the enzymatic hydrolysis of particulate substrates where the loss of small solubilized particles produced by the enzymatic attacks has to be taken into account (Lebaz et al. 2015). To address this issue, different numerical techniques have been proposed for the reconstruction of the PDF from its moments reviewed by John et al. (2007), mainly for the Hausdorff moment problem since in chemical engineering applications, the support of the PDF is known in most cases.

From a mathematical point of view, the moment problem has been extensively studied focusing on the conditions of existence of a unique or infinite solution(s) (Shohat \& Tamarkin, 1943; Akhiezer, 1965; Dette, 1997). Theoretically, a perfect reconstruction can be obtained using an infinity of PDF moments with an $a$ priori restriction of the class of basis functions (John et al., 2007). Numerically, this problem is known as a difficult inverse problem since the finite number of moments define a high ill-posed system of equations (Athanassoulis \& Gavriliadis, 2002). Thus, there is no absolute method for reconstructing accurately the PDF from a finite number of its moments. From an experimental point of view the number of moments that can be obtained with a reasonable accuracy is limited.

The most intuitive reconstruction technique is to approximate the target PDF by a sum of elementary distributions (e.g. Gaussian, log-normal) (Lee, 1983 Diemer \& Olson, 2002) when an information on the shape of the PDF is available a priori. Thus, the problem is reduced to a simple parameter fitting. This technique offers some advantages like the reduced number of moments required for the reconstruction, its simplicity and fastness but the shape of the target PDF has to be stipulated. This constitutes its principal limitation when the initial shape of the PDF changes dramatically during the course of the process. Inspired by this 
approach, Athanassoulis \& Gavriliadis (2002) proposed a robust technique based on the PDF approximation by a finite superposition of kernel density functions (KDF) for the Hausdorff moment problem, tested and validated successfully against both monomodal and bimodal PDFs. This approach has been extended for the Stieltjes moment problem using a generalized Gamma function as the kernel density function (Gavriliadis \& Athanassoulis, 2012, 2009) but unfortunately it was not confronted to realistic cases.

John et al. (2007) proposed an innovative technique using a piecewise polynomial function without any $a$ priori assumption about the shape of the target PDF. Linear, quadratic or cubic splines can be used, their coefficients are computed by solving an ill-conditioned linear system of equations. De Souza et al. (2010) improved the spline-based method by developing an adaptive algorithm in order to optimize the distribution of the grid nodes and capture more accurately the PDF in critical domains. This technique has been coupled recently with a PBE describing aggregation of urea particles (Hackbusch et al., 2012), droplet coalescence (Bordás et al. 2012) and pharmaceutical drying process (granules) (Mortier et al., 2014).

The third class of methods that will be considered in this paper is the Maximum entropy (ME) method which received increasing interest in the last two decades(Mead \& Papanicolaou, 1984, Tagliani, 1999, 2001, Biswas \& Bhattacharya, 2010). This technique is based on the maximization of the Shannon entropy from information theory, by solving a constraint optimization problem. Abramov (2006) extended it to multidimensional problems. Massot et al. (2010) coupled this technique with DQMOM in the case of droplet evaporation process.

In this paper we propose firstly an assessment of these methods in terms of accuracy, rapidity and minimum number of moments required for the reconstruction. The issue concerns the identification of the most efficient method to perform the reconstruction throughout the process (not only for a particular distribution). The performances of the three methods will be evaluated through a comparison of the reconstructed PDFs with an analytical solution of the PBE accounting for a breakage process. Finally, the implementation of the relevant method in the QMOM code for a simultaneous resolution of the PBE and reconstruction of the PDF is investigated. The outlines of this contribution are schematically summerized in figure 1

\section{Reconstruction methods}

The purpose of the reconstrution techniques is to recover a function $f(x)$, given its integer moments sequence $\mu_{n}$ defined as:

$$
\mu_{n}=\int x^{n} f(x) d x, \quad n=0,1, \ldots
$$

We give hereafter a succinct description of the reconstruction techniques used in this contribution. For more detail, one can refer to the original works cited and/or to Appendix A. As common point, the bounded support $[\mathrm{a}, \mathrm{b}]$ is rescaled to $[0,1]$ for a general formulation. 


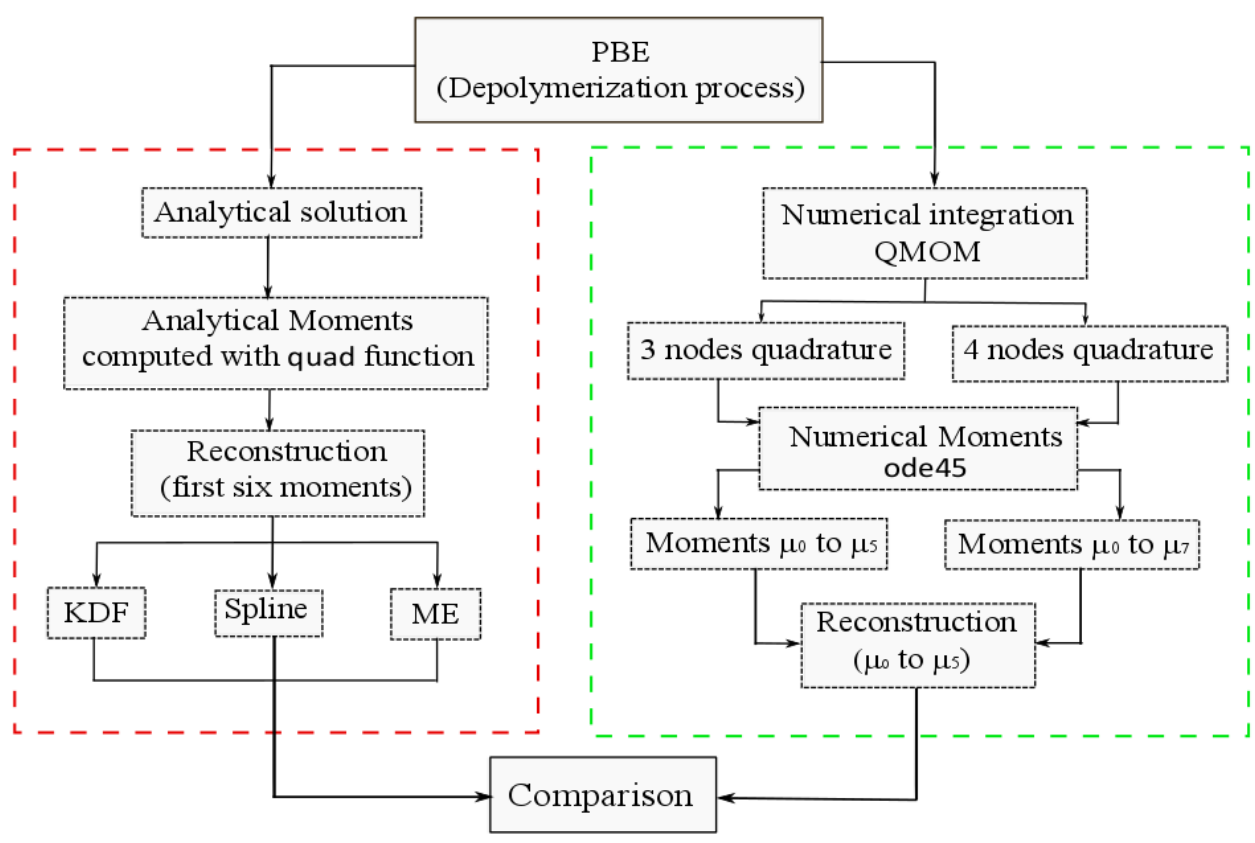

Figure 1: Schematic representation of the organigram of the contribution

\subsection{Kernel Density Function-based method}

In the KDF based technique, the target PDF is approximated by a sum of weighted kernel density functions (equation 2) (Athanassoulis \& Gavriliadis, 2002):

$$
f(x)=\sum_{i=1}^{I} p_{i} K\left(x ; x_{i}, h\right)
$$

where $K\left(x ; x_{i}, h\right)$ are the KDFs, centered at $x_{i}$, with bandwidth $h, I$ is the total number of kernel density functions used for the reconstruction. The coefficients $p_{i}$ satisfy:

$$
p_{i} \geq 0, \quad i=1,2, \ldots, I ; \quad \sum_{i=1}^{I} p_{i}=1
$$

Since the target PDF is univariate, the KDF is chosen as a Beta Kernel. Thus, the objective is the determination of the optimal coefficients $p_{i}$. For this, the finite-moment problem is reformulated as a constrained optimization problem aiming to find the coefficients $p_{i}$ which minimize the error between the set of initial moments and those estimated via the sum of Beta KDFs. Numerically, Nonnegative Least Square algorithm (NNLS) can be used in this case. Optimization techniques are to be supplemented in order to improve the efficiency of this procedure. The properties of the Beta Kernel Function, the formulation of the constrained optimization problem, the optimization technique based on the shifted moments and the general algorithm 
of this method are described in Appendix A.1

\subsection{Spline-based method}

This method is extensively described by John et al. (2007). The support of the target PDF $[a, b]$ is subdivided into $n$ subintervals such as $a=x_{1}<x_{2}<\cdots<x_{n+1}=b$. In each subinterval $\left[x_{i}, x_{i+1}\right]$, the target PDF is approximated by a piecewise polynomial $s^{(l)}(x)$ of degree $l$. The system of equations is detailed for cubic splines $(l=3)$. The unknowns are the four coefficients of the $n$ splines. A smooth transition at the boundaries of the interval is assumed meaning that the PDF, its first and second derivatives are null at the boundaries, this gives $2 \times 3$ equations. The continuity of the splines, their first and second derivatives at the nodes provides $3(n-1)$ equations. The remaining $(n-3)$ equations are supplemented by the moments. This leads to solve a $4 n \times 4 n$ ill-conditioned linear system.

Since the set of the known moments limits the number of splines, one has to compute the reconstruction in the optimal support of $f(x)$ thus, the reconstruction is computed iteratively starting from an initial reconstruction $f^{(0)}(x)$ in an initial interval $\left[x_{1}^{(0)}, x_{n+1}^{(0)}\right]$. To ensure the positivity of the reconstructed PDF a tolerance $t_{\text {neg }}$ is introduced. Furthermore, the optimization of the distribution of the nodes improves the efficiency of the method especially for multimodal functions. More details on these techniques are given in Appendix A.2

\subsection{Maximum Entropy method}

The Maximum Entropy method is based on the maximization of the Shannon entropy $H[f]$ from information theory given by:

$$
H[f]=-\int_{0}^{\infty} f(x) \ln f(x) d x
$$

Under the moments constraints defined by equation 1.

The explicit representation of the ME approximation $f_{M}(x)$ of the target PDF takes the form:

$$
f_{M}(x)=\exp \left[-\sum_{j=0}^{N} \xi_{j} x^{j}\right]
$$

To be supplemented by the $(N+1)$ constraints:

$$
\mu_{i}=\int_{0}^{\infty} x^{i} f_{M}(x) d x \quad i=0,1, \ldots, N
$$

The $(N+1)$ Lagrange's multipliers $\xi_{0}, \ldots, \xi_{N}$ are obtained through the resolution of the following set of $(N+1)$ nonlinear equations:

$$
\int_{0}^{\infty} x^{i} \exp \left[-\sum_{j=0}^{N} \xi_{j} x^{j}\right] d x=\mu_{i} \quad i=n, 1, \ldots, N
$$


Numerically, iterative methods are used to solve equation 7 (Mead \& Papanicolaou, 1984). In our case,

115

a given tolerance for stopping the iterative procedure. Commonly used parameters are given in the results section.

\section{Population balance modelling (PBM)}

The mathematical modelling of polymer chain fragmentation processes (thermal, thermochemical and bio-

logical) is a fundamental issue in polymer science and engineering (McCoy \& Madras, 2001; Bose \& Git, 2004). Population balance modelling (PBM) is the classical approach since it describes the dynamical evolution of the Chain Length Distributions (CLD) (or MWD: Molecular Weight Distributions) during depolymerization reactions (Ziff \& McGrady, 1985, Madras \& McCoy, 1998).

For a homogeneous system, the time evolution of the CLD undergoing fragmentation processes is described by the population balance equation (PBE) below, written in its continuous form:

$$
\frac{\partial n(L, t)}{\partial t}=\int_{L}^{\infty} \beta\left(L, L^{\prime}\right) \gamma\left(L^{\prime}\right) n\left(L^{\prime}, t\right) d L^{\prime}-\gamma(L) n(L, t)
$$

where $n(L, t)$ is the length-based number density function (CLD), $\gamma(L)$ the breakage frequency for a chain of length $L, \beta\left(L, L^{\prime}\right)$ is the breakage kernel giving the probability of obtaining a chain of length $L$ from the breakup of a chain of length $L^{\prime}$. The first term on the RHS accounts for the formation (birth) of chains with length $L$ resulting from the breakage of longer chains $L^{\prime}$. The last term is the death term due to the loss of chains of length $L$ because of their breakup.

Except for some cases where analytical solutions exist, equation 8 is solved numerically. Since the resolution of this equation is not the objective of this contribution, an analytical solution is briefly described and used as reference for the moment problem.

\subsection{Analytical solution of the PBE accounting for breakage process}

135

Ziff \& McGrady (1985) considered linear polymers of length $L$ and gave the analytical solution of the equation 8 with the breakage kernel $\beta=2 / L$ and the breakage frequency $\gamma=\alpha L^{2}$ ( $\alpha$ is a factor of proportionality) which can be regarded as the number of attacks per time unit. Under these considerations, equation 8 can be rewritten as:

$$
\frac{\partial n(L, t)}{\partial t}=2 \alpha \int_{L}^{\infty} L^{\prime} n\left(L^{\prime}, t\right) d L^{\prime}-\alpha L^{2} n(L, t)
$$

The general solution of the equation 9 is given as (Ziff \& McGrady, 1985):

$$
n(L, t)=e^{-\alpha t L^{2}}\left(n(L, 0)+2 \alpha t \int_{L}^{\infty} L^{\prime} n\left(L^{\prime}, 0\right) d L^{\prime}\right)
$$


where $n(L, 0)$ is the initial CLD.

In order to explicit this solution, we assume that the initial chain length distribution (CLD) follows a normal law (equation 11) with $(m, \sigma)$ its mean and standard deviation respectively.

$$
n(L, 0))=\frac{1}{\sigma \sqrt{2 \pi}} e^{-\frac{(L-m)^{2}}{2 \sigma^{2}}}
$$

The explicit solution in this specific case is given as (details are given in Appendix B) :

$$
n(L, t)=\frac{1}{\sigma \sqrt{2 \pi}} e^{-\alpha t L^{2}}\left[\left(2 \alpha t \sigma^{2}+1\right) e^{-\frac{1}{2}\left(\frac{L-m}{\sigma}\right)^{2}}+m \sigma \alpha t \sqrt{2 \pi}\left(1-\operatorname{erf}\left(\frac{1}{\sqrt{2}} \frac{L-m}{\sigma}\right)\right)\right]
$$

where $\operatorname{erf}$ refers to the error function.

145

The Quadrature Method of Moments (QMOM) is based on the Gaussian quadrature of the continuous CLD (McGraw, 1997):

$$
n(L, t) \approx \sum_{i=1}^{M} w_{i}(t) \delta\left(L-L_{i}(t)\right)
$$

where $M$ is the number of nodes $i, L_{i}$ is the property of the node (length), $w_{i}$ its weight, and $\delta$ is the Dirac function. Thus, the $k^{\text {th }}$ moment can be expressed as :

$$
\mu_{k}(t)=\int_{0}^{\infty} n(L) L^{k} d L \approx \sum_{i=1}^{M} w_{i}(t) L_{i}(t)
$$

where weights $\left(w_{i}\right)$ and abscissas $\left(L_{i}\right)$ are determined through the Product-Difference algorithm (Gordon, 1968). By substituting equations 14 and 15 in 13 , we obtain (Marchisio et al., 2003b) :

$$
\frac{\partial \mu_{k}}{\partial t}=\sum_{i=1}^{M} \gamma_{i} \bar{b}_{i}^{(k)} w_{i}-\sum_{i=1}^{M} L_{i} \gamma_{i} w_{i}
$$




$$
\bar{b}_{i}^{(k)}=\int_{0}^{\infty} L^{k} \beta\left(L, L_{i}\right) d L
$$

Generaly, the QMOM requires at least a three nodes quadrature $(M=3)$ for an accurate time-tracking of the moments (Marchisio et al., 2003b).

MATLAB is used under a work-station comprising of an Intel ${ }^{\circledR}$ Core $^{\mathrm{TM}_{\mathrm{i}} \text { 7-3740QM }} \mathrm{CPU}$ with a clock speed of $2.7 \mathrm{GHz}$ and $16 \mathrm{~GB}$ of installed memory (RAM).

\subsection{Reconstruction of the analytical solution}

As described before, the three reconstruction methods will be compared to the analytical solution given in section 3-1. We assume that the initial CLD is given by a normal law centered at a Degree of Polymerization (DP) of 60 (the mean number of monomers constituting a given polymer) with a standard deviation $(\sigma)$ of in figure 2 with $\alpha=10^{-3}$ and $t \in\left[t_{i}, t_{f}\right]=[0,1]$.

Figure 2 shows a rapid degradation of the long polymers since the breakage frequency is proportional to $L^{2}$, the concentration of the small polymer chains increases all along the depolymerization process.

For sake of clarity, the ability of the different methods to reconstruct the time evolution of the CLD is $t_{4}=t_{f}$ before addressing a comparative analysis. At any given instant, the reconstruction is initiated by computing the set of the moments of the analytical CLD given in equation (12). The integrations leading to the associated moments, equation (1), are performed using the Matlab function quad with a tolerance set to $10^{-6}$. Since the number of the moments required for the reconstruction is critical, the methods are tested with the same set of moments transformed systematically to the normalized interval $[0,1]$ before rescaling the reconstruction result (figure11). 


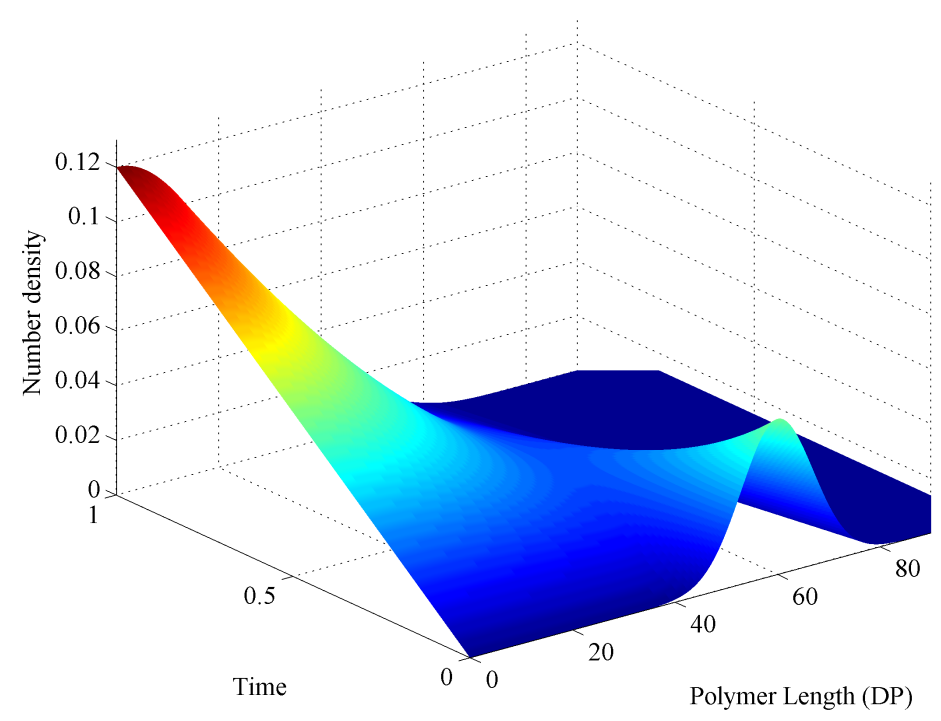

Figure 2: Time evolution of the CLD given by the analytical solution of Ziff \& McGrady (1985)

In order to compare the different reconstruction methods, we introduce the mean absolute error between the reconstructed CLD $\left(f_{M}(x)\right)$ and the analytical solution used as a reference $(f(x))$ :

$$
\operatorname{Er}(t)=\frac{1}{q} \sum_{i=1}^{q}\left|f(x, t)-f_{M}(x, t)\right|
$$

where $q$ is the length of the discretized support.

\subsubsection{Kernel Density Function-based method}

The algorithm of this method was implemented in Matlab using the set of moments of each target CLD with their associated shifted moments by setting $\bar{x}=0.8$ in order to increase the number of the constraints and thus improve the efficiency of this technique. The number of Beta Kernel Density Functions (BKDFs) $I$ is set to 15 thus, the support [0,1] is divided into 15 equal intervals, each BKDF is centered on the midpoint of its corresponding interval. Athanassoulis \& Gavriliadis (2002) showed that the optimal bandwidth parameter $h$ varies in the interval $[0.04,0.09]$. In order to identify the optimal values for $h$, the interval is scanned and the reconstruction is conducted at different parameter values before selecting the most accurate. Once the BKDFs parameters are fixed, the associated Beta PDF parameters are calculated and the coefficients $B_{n, i}$ and $\tilde{B}_{n, i}$ (equations A.3 and A.8 are computed. The constraint minimization problem (equation A.10 is solved using LSQLIN subroutine.

Figure 3 shows the results for the five different instants using the first six moments of each target CLD.

Although the initial CLD is reconstructed with great accuracy most probably because of its simple form, the other CLDs are not recovered. Their singular shape mainly around the origin makes their reconstruction challenging. The reconstructions are oscillating around the target CLDs and take the value zero at the origin. 


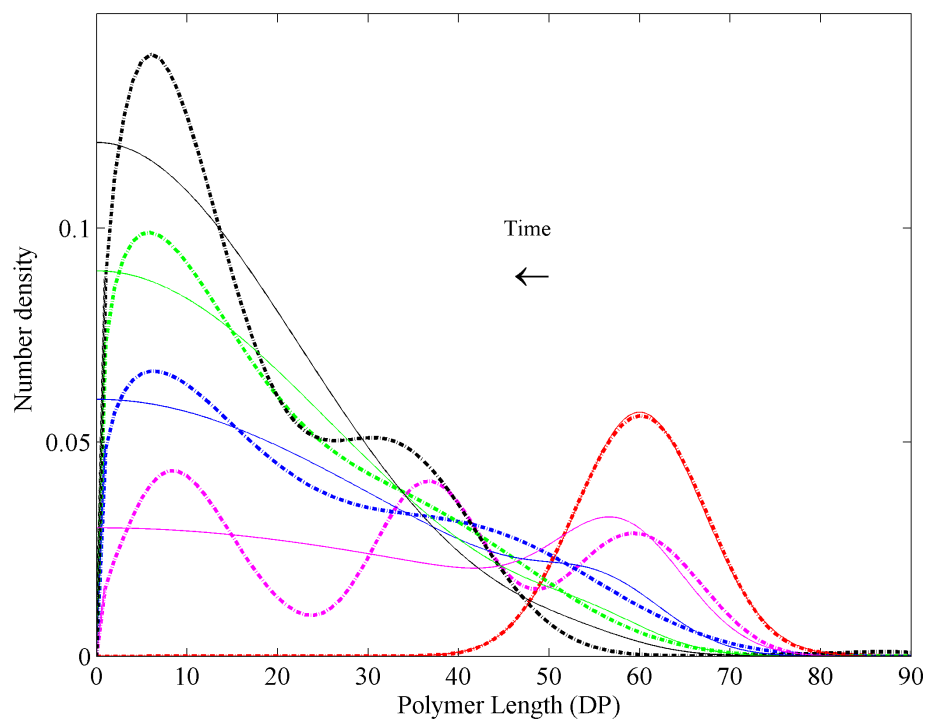

Figure 3: Comparison between the target CLDs (continuous line) and their reconstructions (dashed line) using the BKDF based technique with a sequence of six analytical moments

To be more accurate, this technique requires a large number of constraints which means a higher number of moments. In figure 4, the same problem is treated using the first eleven moments of each target CLD.

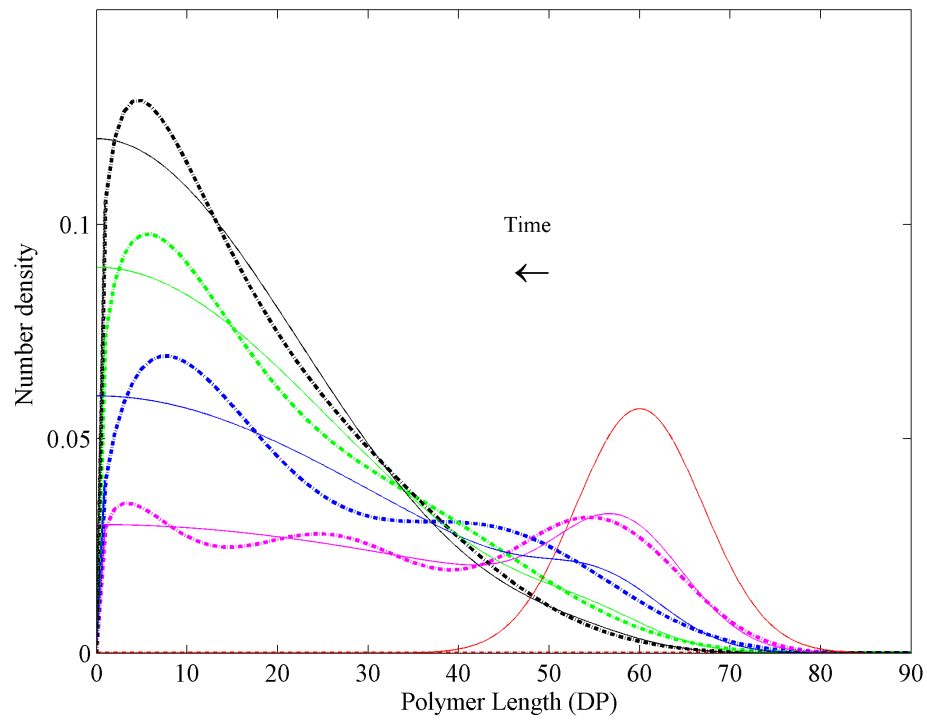

Figure 4: Comparison between the target CLDs (continuous line) and their reconstructions (dashed line) using the BKDF based technique with a sequence of eleven analytical moments

The figure 4 shows that the use of a high number of moments can improve the reconstruction quality 
as illustrated in Table 1 where the mean absolute errors (equation 18) are calculated in both cases. But, since the technique is treating a highly ill-posed problem, the solution of the constraint system of equations is attempted (figure 4). To sum up, this method requires a finetuning of the numerical parameters and an increase in the number of moments does not always guaranties an easier reconstruction. However if the reconstruction is successful, the accuracy increases with the number of moments.

\begin{tabular}{|c|c|c|}
\hline \multirow{2}{*}{ Time } & \multicolumn{2}{|c|}{ Mean absolute error } \\
\cline { 2 - 3 } & BKDF: 6 moments & BKDF: 11 moments \\
\hline$t_{0}$ & 0.0002 & $/$ \\
\hline$t_{1}$ & 0.0061 & 0.0014 \\
\hline$t_{2}$ & 0.0026 & 0.0033 \\
\hline$t_{3}$ & 0.0028 & 0.0024 \\
\hline$t_{4}$ & 0.0068 & 0.0023 \\
\hline
\end{tabular}

Table 1: Reconstruction accuracy using the BKDF based technique with 6 and 11 moments

\subsubsection{Spline-based method} This is not reachable without an a priori information on the shape of the target PDF. 


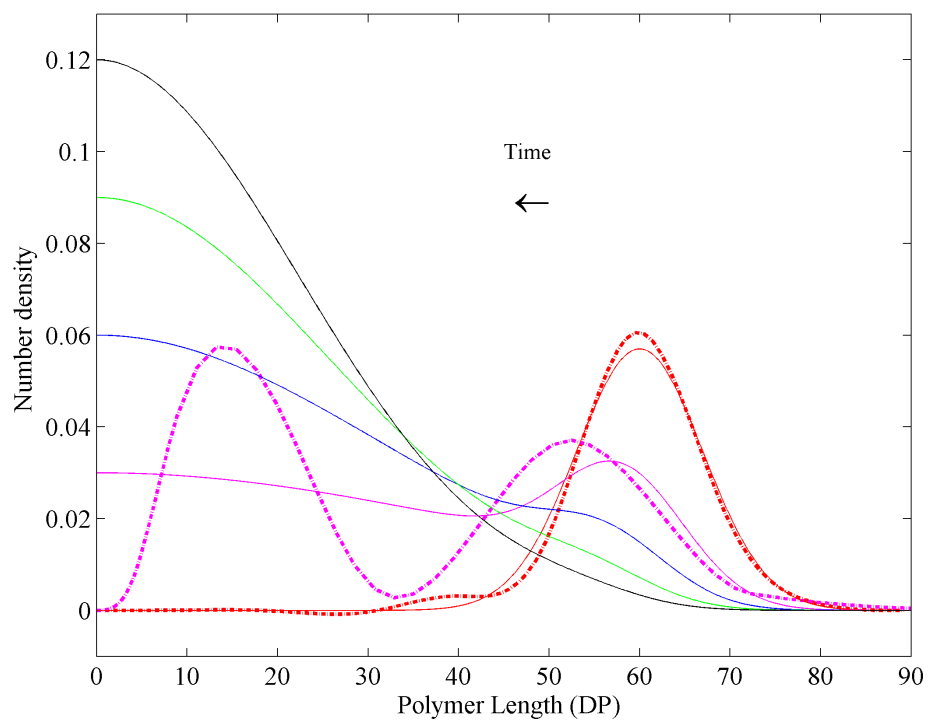

Figure 5: Comparison between the target CLDs (continuous line) and their reconstructions (dashed line) using the spline based technique with a sequence of six analytical moments

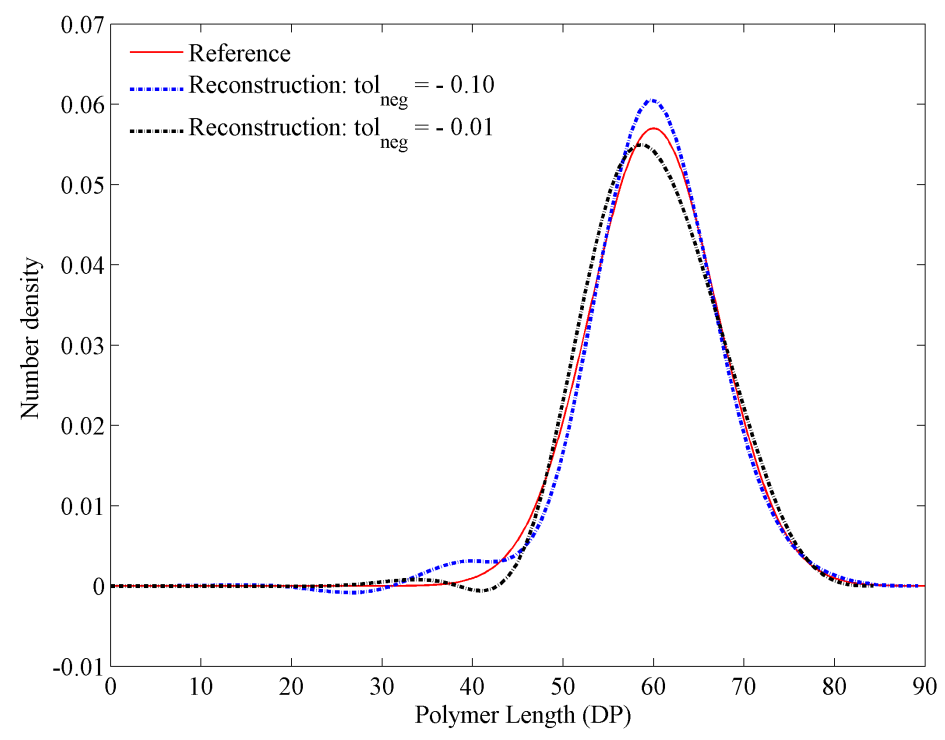

Figure 6: The sensitivity of the spline based method to $t_{\text {neg }}$

\subsubsection{Maximum Entropy based method}

The non-linear system in equation 7 is solved using Newton method starting from an initial choice of the

Lagrange's multipliers as $\xi^{(0)}=\left(-\ln \left(\mu_{0}\right) /\left(x_{\max }-x_{\min }\right), 0, \ldots, 0\right)$ with a tolerance of $10^{-6}$ (Massot et al. 2010). The moments are scaled to $[0,1]$ and normalized before such as $\mu_{0}=1$. The results are given in figure 


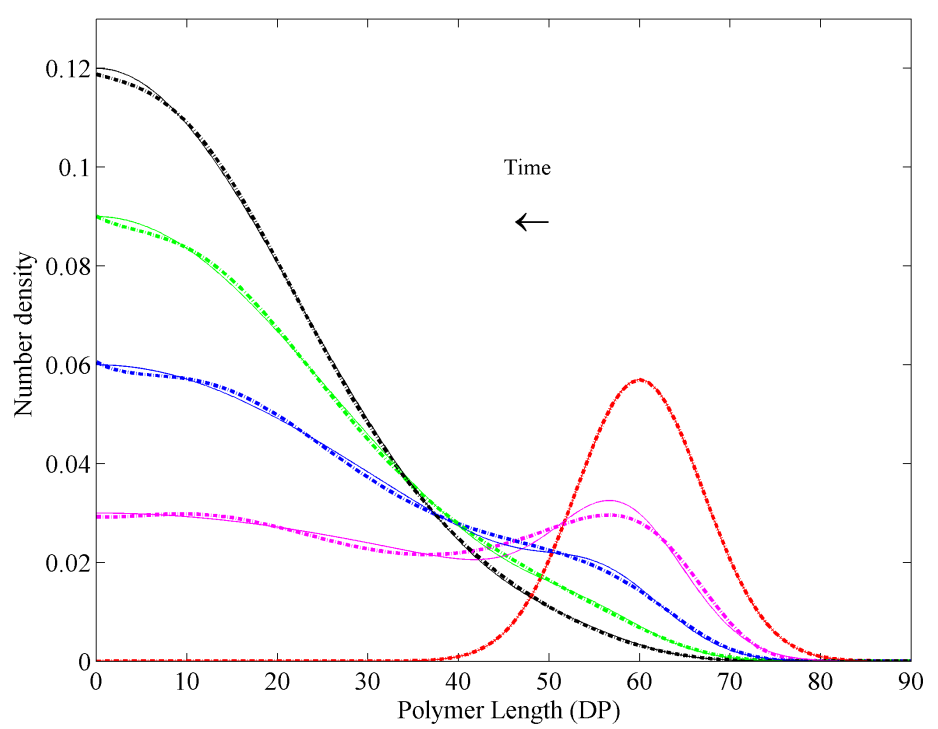

Figure 7: Comparison between the target CLDs (continuous line) and their reconstructions (dashed line) using the ME based technique with a sequence of six analytical moments

Unlike the two previous methods, the Maximum Entropy technique reconstructs the analytical CLDs with high accuracy even when they are singular using only the first six moments. The Newton method used in this case converges in a few iterations $(<10)$ making the coupling of the PBE resolution with the PDF reconstruction possible. In addition, except for the tolerance of the Newton method, there is no additional parameter to set which simplifies its use.

\begin{tabular}{|c|c|c|c|c|c|c|}
\hline \multirow{2}{*}{ Time } & \multicolumn{2}{|c|}{ BKDF based method } & \multicolumn{2}{c|}{ Spline based method } & \multicolumn{2}{c|}{ ME based method } \\
\cline { 2 - 7 } & Error & Cost $(\mathrm{s})$ & Error & Cost $(\mathrm{s})$ & Error & Cost $(\mathrm{s})$ \\
\hline$t_{0}$ & 0.0002 & 6.48 & 0.0011 & 6.48 & 0.0000 & 0.27 \\
\hline$t_{1}$ & 0.0061 & 6.47 & 0.0105 & 5.86 & 0.0008 & 0.26 \\
\hline$t_{2}$ & 0.0026 & 6.39 & $/$ & $/$ & 0.0005 & 0.23 \\
\hline$t_{3}$ & 0.0028 & 6.31 & $/$ & $/$ & 0.0004 & 0.20 \\
\hline$t_{4}$ & 0.0068 & 6.30 & $/$ & $/$ & 0.0004 & 0.20 \\
\hline
\end{tabular}

Table 2: Comparison of the three different reconstruction methods (using the first six analytical moments) in terms of mean absolute error and computational cost

Table 2 gives the comparison between the three methods according to the quality of the reconstructed CLDs (mean absolute error) and the computational cost using the first six moments. The ME method gives 

of moments required and the computational cost among other criterions as resumed in Table 3 For these reasons, this method is selected to be implemented in the QMOM code for coupling the PBE resolution in the case of depolymerization process with the CLD reconstruction.

\begin{tabular}{|c|c|c|c|}
\hline Criterion & Beta KDF method & Spline-based method & ME based method \\
\hline Principle & $\begin{array}{l}\text { Approximation via a sum } \\
\text { of kernel density functions }\end{array}$ & $\begin{array}{l}\text { Approximation via con- } \\
\text { nected peicewise polyno- } \\
\text { mials }\end{array}$ & $\begin{array}{l}\text { Approximation based on } \\
\text { the maximization of Shan- } \\
\text { non entropy }\end{array}$ \\
\hline Target PSD & $\begin{array}{l}\text { No a priori information } \\
\text { on its shape }\end{array}$ & $\begin{array}{l}\text { No a priori information } \\
\text { on its shape }\end{array}$ & $\begin{array}{l}\text { No a priori information } \\
\text { on its shape }\end{array}$ \\
\hline $\begin{array}{l}\text { Number of mo- } \\
\text { ments }\end{array}$ & $>10$ to be accurate & $\begin{array}{l}\text { Related to the number of } \\
\text { intervals }\end{array}$ & Six or more \\
\hline Computational cost & Acceptable & $\begin{array}{l}\text { Acceptable when it con- } \\
\text { verges }\end{array}$ & Very low \\
\hline Robustness & $\begin{array}{l}\text { The convergence is not } \\
\text { guaranteed in all cases }\end{array}$ & $\begin{array}{l}\text { The convergence is highly } \\
\text { dependent on the parame- } \\
\text { ters of the method }\end{array}$ & $\begin{array}{l}\text { The convergence is guar- } \\
\text { anteed in most cases }\end{array}$ \\
\hline $\begin{array}{l}\text { Accuracy of the re- } \\
\text { construction }\end{array}$ & $\begin{array}{l}\text { Depends on the number of } \\
\text { moments, acceptable for } \\
\text { simple shapes and oscil- } \\
\text { lates for complex shapes }\end{array}$ & $\begin{array}{l}\text { Highly oscillating results } \\
\text { even for simple shapes, de- } \\
\text { pends on the parameters } \\
\text { of the method }\end{array}$ & $\begin{array}{l}\text { Good occuracy even with } \\
\text { just six moments, not af- } \\
\text { fected by the parameters } \\
\text { initialisation }\end{array}$ \\
\hline
\end{tabular}

Table 3: General comparison between the three different reconstruction methods

\subsection{QMOM coupled with ME based method}

The main objective of the development of reconstruction methods from a finite set of moments, especially in the chemical engineering field, is to have access to the shape of the PSD and/or some relevant pointwise values during the process evolution. This kind of information is necessary when some aspects of the problem cannot be expressed from the moments only. This offers a valuable addition when the PBE is solved using moment methods coupling by the fact the computational efficiency of such methods with a simultaneous

In this section, the ME based method tested successfully against an analytical solution giving the time evolution of the CLD undergoing breakage process is implemented in the QMOM code resolving the same breakage problem. Thus, on the one hand, the analytical CLDs are available for comparison, on the other 
hand, the PBE accounting for the same breakage process is solved using QMOM giving the time evolution of the moments which are used for the reconstruction (see figure 1) .

For this numerical approach, a three nodes quadrature is used $(\mathrm{M}=3)$, the abscissas $\left(L_{i}\right)$ and the weights $\left(w_{i}\right)$ are computed using the Product-Difference algorithm (Gordon, 1968). The system of ordinary differential equations (equation 16 ) is integrated using ode 45 with a tolerance fixed to $10^{-6}$. First, the QMOM implementation is validated by confronting the computed moments against those predicted by the analytical solution.
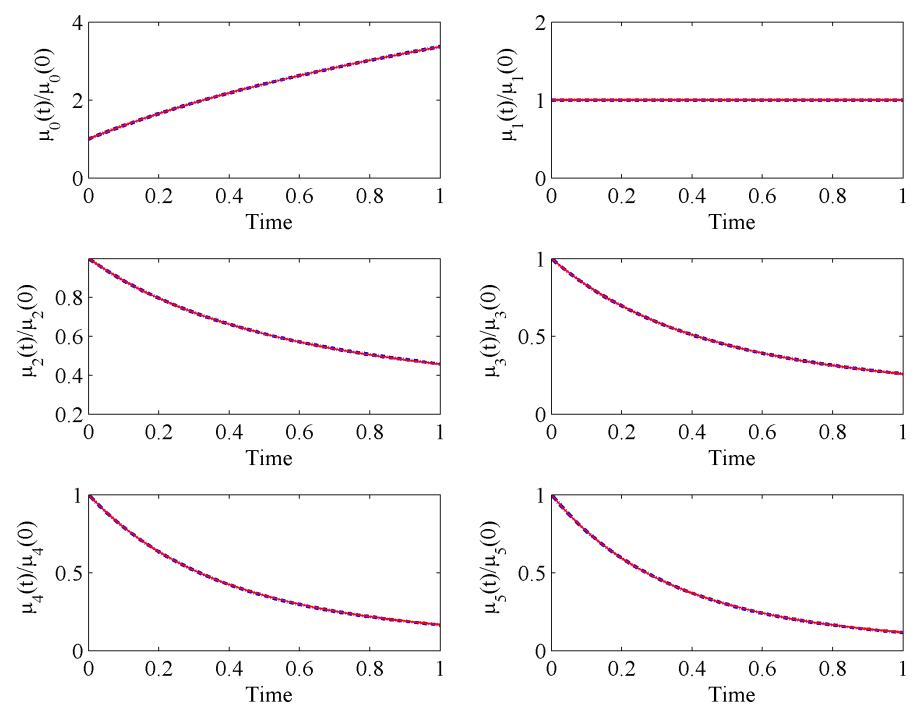

Figure 8: The time evolution of the six first moments: comparison between the analytical solution (continuous line) and QMOM with three nodes (dashed lines)

The results for the six first moments are given in figure 8 . The two different methods lead to the same time evolution of the moments thus, the QMOM implementation is validated.

Since a three nodes quadrature is used, six moments are tracked. This set of moments is used as input for the ME based method. The reconstruction is conducted simultaneously with the PBE resolution. The result at different process time is shown in figure 9 .

The reconstructions based on the numerical moments computed via QMOM (figure 9) are less accurate than those obtained previously with the exact analytical moments (figure 7). Note that the same number of moments is used in the two cases. This can only comes from the numerical error introduced by QMOM. In order to improve the accuracy of the method, one can increase the number of the quadrature nodes. For quantifying the gain in terms of accuracy, we introduce the global error function below:

$$
E(k)=\frac{\left|\mu_{k}-\mu_{k}^{*}\right|}{\mu_{k}^{*}}
$$




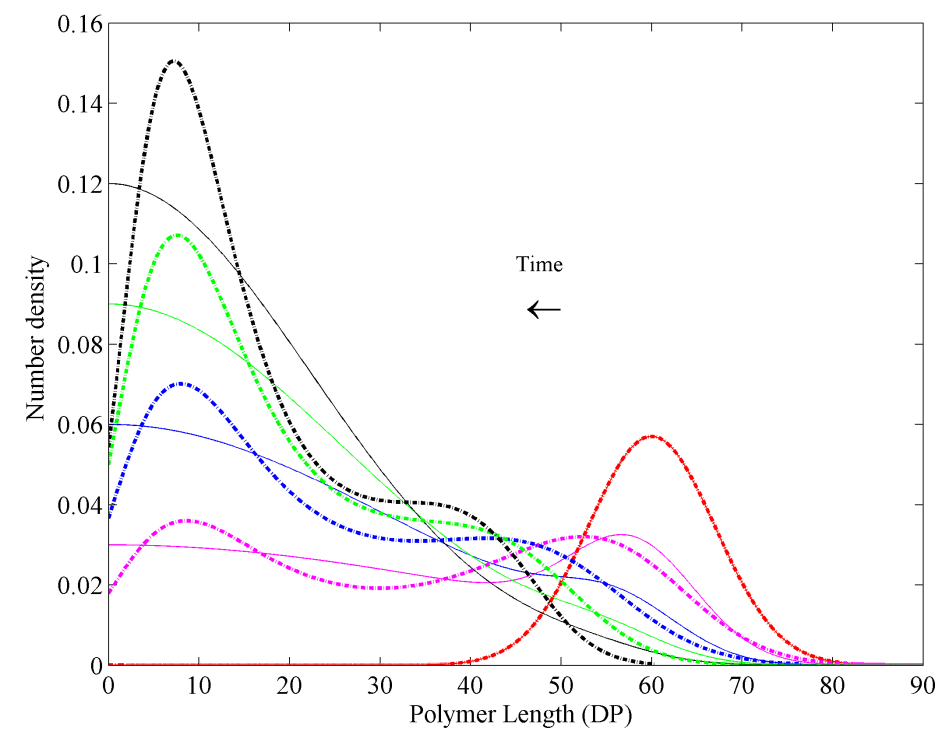

Figure 9: Comparison between the target CLDs (continuous line) and their reconstructions (dashed line) using the ME based technique with a sequence of six moments computed via QMOM with a three nodes quadrature

where $\mu_{k}$ is the $k^{t h}$ order moment estimated via QMOM and $\mu_{k}^{*}$ the exact $k^{t h}$ order moment calculated via the analytical solution.

We give in figure 10 the error $E(k)$ induced by QMOM for both a three and four nodes quadrature for the six first moments. Note that the use of a four nodes quadrature implies a time tracking of the first eight moments of the CLD.

The use of a four nodes quadrature in QMOM improves the accuracy of the computed moments, which consitutes a trivial and expected result. Note however that the use of a higher number of nodes induces supplementary moments meaning more equations in the system (equation 16) and may lead to near-singular matrix limiting the efficiency of PD algorithm.

Now, if the first six moments computed using a four nodes quadrature are used for the reconstruction, the result is completely different as shown in figure 11 and in Table 4

Improving the accuracy of the tracked moments has considerably improved the reconstruction quality without reaching the same performance as with the analytical solution (figure 7). This shows that, if the ME based method is coupled with QMOM, one has to compute the moments with a high accuracy. For the reconstruction purpose, it is wise to track some additional moments in order to improve the accuracy of the set of moments that will be used.

To avoid the use of the PD algorithm for computing the weights $\left(w_{i}\right)$ and the abscissas $\left(L_{i}\right)$ all along the process which may introduce a subsequent error for the computed moments, the Direct Quadrature Method of Moments (DQMOM) presents an alternative (Marchisio \& Fox, 2005). In this method, the PD algorithm soo is only called once, for initializing the quadrature nodes. 

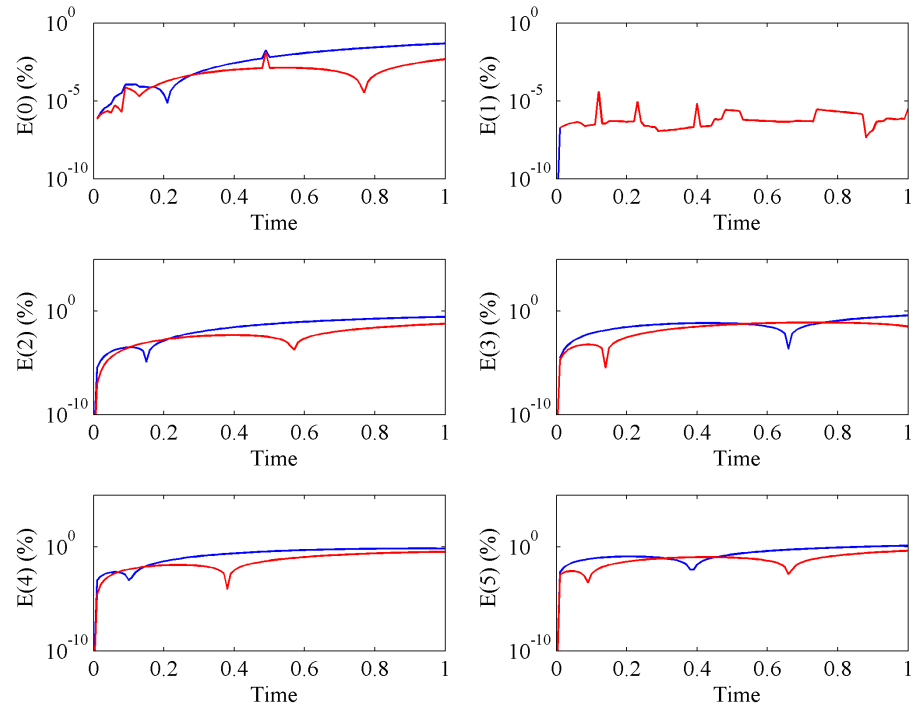

Figure 10: Comparison of the error induced by QMOM for the six first moments when using a three nodes quadrature (blue line) and a four nodes quadrature (red line)

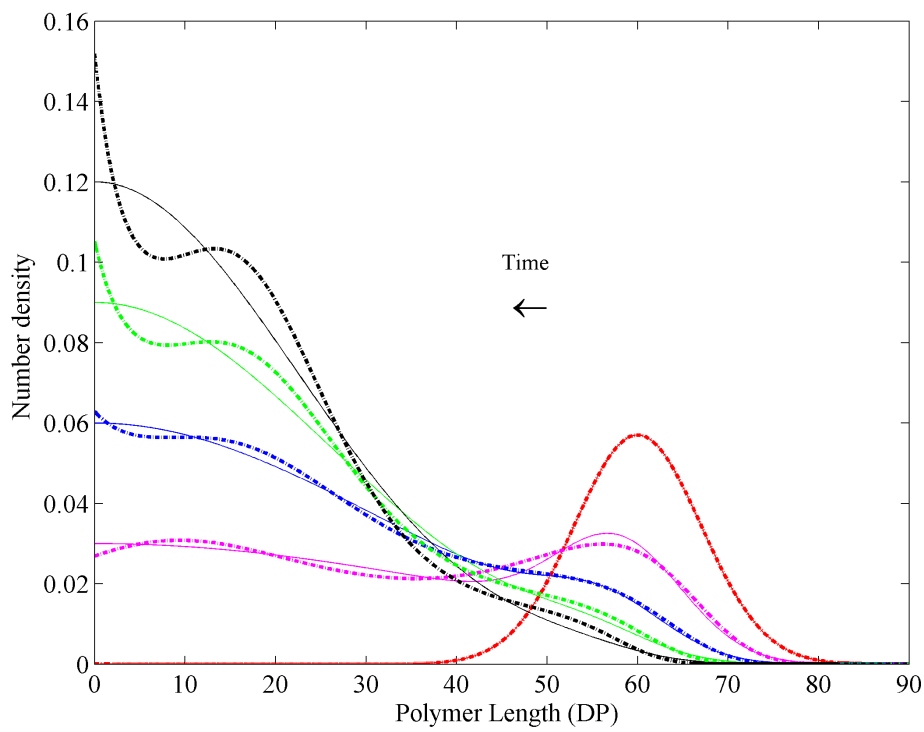

Figure 11: Comparison between the target CLDs (continuous line) and their reconstructions (dashed line) using the ME based technique with a sequence of six moments computed via QMOM with a four nodes quadrature

This method has been investigated and comparative results as with QMOM are obtained. The error on computing the initial $\left(L_{i}, w_{i}\right)$ is transported throughout the process time. In addition, it has been shown that more accurate and robuste algorithms can be used instead of the PD algorithm for computing the nodes (John \& Thein, 2012). The use of the Wheeler algorithm (called also LQMDA for Long Quotient-Modified 


\begin{tabular}{|c|c|c|}
\hline \multirow{2}{*}{ Time } & \multicolumn{2}{|c|}{ Mean absolute error } \\
\cline { 2 - 3 } & QMOM-ME: M=3 & QMOM-ME: M=4 \\
\hline$t_{0}$ & 0.0000 & 0.0000 \\
\hline$t_{1}$ & 0.0027 & 0.0010 \\
\hline$t_{2}$ & 0.0037 & 0.0007 \\
\hline$t_{3}$ & 0.0054 & 0.0019 \\
\hline$t_{4}$ & 0.0080 & 0.0031 \\
\hline
\end{tabular}

Table 4: Reconstruction accuracy using the ME based technique with six moments: comparison between the use of the moments computed with a three nodes quadrature and those obtained with a four nodes quadrature methods. This has been investigated in the second part.

Once the Quadrature Method of Moments accounting for breakage processes is validated against the analytical solution, the computed moments are used as inputs for the reconstruction method. The results 
show that the ME based technique is highly dependent on the moments accuracy. When a three nodes quadrature is used for tracking the abscissas and the weights via the PD algorithm, the sets of associated moments lead to unsatisfactory reconstructions. Increasing the number of nodes improves significantly the moments accuracy and by the way the quality of the reconstruction. No doubt that the issue is common to all reconstruction methods: accurate values of the moments are required.

In summary, the ME based method is preferred than the BKDF and spline based methods because it requires a smaller number of moments and offers the best computational cost/reconstruction accuracy. The cases where a frequent reconstruction is needed along with the PBE resolution, the moments have to be computed accurately to acheive a good reconstruction quality using the ME based method. This is the main drawback when dealing with experimental moments. Further confrontation especially when different processes are coupled (e.g. breakage, aggregation, growth) is needed for assessing the relevance of this method.

\section{Aknowledgments}

The authors would like to thank Toulouse White Biotechnology and Région Midi-Pyrénées for their financial support.

\section{Appendix A: Description of the reconstruction techniques}

Appendix A.1: Kernel Density Function-based method

The Beta PDF is given, in its normalized form with $\kappa$ and $\lambda$ as parameters, by:

$$
g(x ; \kappa, \lambda)=\frac{\Gamma(\kappa+\lambda)}{\Gamma(\kappa) \Gamma(\lambda)} x^{\kappa-1}(1-x)^{\lambda-1}, \quad \kappa, \lambda>0
$$

where $\Gamma$ is the Gamma function. The Beta kernel is then defined by :

$$
K\left(x ; x_{*}, h\right)=g(x ; \kappa, \lambda)
$$

The Beta KDF parameters $\left(x_{*}\right.$ and $h$ ) are related to the Beta PDF parameters $(\kappa$ and $\lambda$ ) (see Appendix A (Athanassoulis \& Gavriliadis, 2002)).

The moments of the Beta kernel can be analytically calculated thanks to the equation A.3:

$$
B_{n}\left(x_{*}\right)=B_{n}(\kappa, \lambda)=\int_{0}^{1} x^{n} g(x ; \kappa, \lambda) d x=\frac{\Gamma(\kappa+\lambda) \Gamma(\kappa+n)}{\Gamma(\kappa) \Gamma(\kappa+\lambda+n)}
$$

By substituting equation A.3 in 2, one obtains the moments $\mu_{n}^{*}$ of the reconstructed distribution :

$$
\mu_{n}^{*}=\sum_{i=1}^{I} B_{n, i} p_{i}, \quad n=0,1, \ldots N_{1}
$$


For determining the unknown coefficients $p_{i}$, the finite-moment problem is reformulated as a constrained optimization problem (equation A.5):

$$
\left\|\mu_{n}-\mu_{n}^{*}\right\|_{L^{2}}=\min , \quad\left(p_{i} \geq 0, \sum_{i=1}^{I} p_{i}=1\right)
$$

Equation A.5 can be solved numerically using a Nonnegative Least Square algorithm (NNLS). For improving the efficiency of this method and reducing the number of moments required for the reconstruction, different optimization techniques are available in literature such as the use, in addition of the given moments, of the shifted moments as described in the original work by (Gavriliadis \& Athanassoulis, 2003).

The shifted moments of $f(x)$ are defined by:

$$
\tilde{\mu}_{n}=\int_{0}^{1}(x-\bar{x})^{k} f(x) d x, \quad n=1,2 \ldots N_{2} \leq N_{1}, \quad \bar{x} \in[0,1]
$$

Since, in the finite-moment problem, $f(x)$ is the target, the shifted moments $\tilde{\mu}_{n}$ are derived from the integer moments $\mu_{n}$ as follows:

$$
\tilde{\mu}_{n}=\sum_{k=0}^{n}(-1)^{k}\left(\begin{array}{l}
n \\
k
\end{array}\right) \bar{x}^{k} \mu_{n-k}, \quad n=1,2 \ldots N_{1} \leq N
$$

The shifted moments of the Beta KDF are calculated by:

$$
\tilde{B}_{n}=\int_{0}^{1}(x-\bar{x})^{n} K\left(x ; x_{i}, h\right) d x
$$

Thus, the shifted moments $\tilde{\mu}_{n}^{*}$ of the reconstructed PDF are expressed as:

$$
\tilde{\mu}_{n}^{*}=\sum_{i=0}^{I} \tilde{B}_{n i} p_{i}, \quad n=1,2 \ldots N_{1} \leq N
$$

The minimization problem (equation A.5) is reformulated as :

$$
\left\|\left(\begin{array}{c}
\mu_{n} \\
\tilde{\mu_{n}}
\end{array}\right)-\left(\begin{array}{c}
B_{n} \\
\tilde{B}_{n}
\end{array}\right) p_{I}\right\|_{L^{2}}=\min , \quad\left(p_{i} \geq 0, \sum_{i=1}^{I} p_{i}=1\right)
$$

This new constraint minimization problem is numerically solved using NNLS or LSQLIN (Matlab subroutines).

The algorithm of this method can be reduced to the following steps:

1. Define the reconstruction interval $[\mathrm{a}, \mathrm{b}]$

2. Transform the moments to the normalized interval $[0,1]$

3. Compute the shifted moments from the set of integer moments

4. Fix the number $I$ of KDFs and the bandwidth parameter $h$

5. Compute the coefficients $B_{n, i}$ and $\tilde{B}_{n, i}$

6. Determine $p_{i}$ by solving the constraint minimization problem (equation A.10 


\section{Appendix A.2: $\quad$ Spline-based method}

In order to optimize the reconstruction support and thus increase the accuracy of the spline-based method, the values of the reconstructed PDF $f^{(k)}(x)$ are checked at the boundaries of the support $\left[x_{1}^{(k)}, x_{n+1}^{(k)}\right]$ (i.e. in the subintervals $\left[x_{1}^{(k)}, x_{2}^{(k)}\right]$ and $\left.\left[x_{n}^{(k)}, x_{n+1}^{(k)}\right]\right)$. For this, these two subintervals are divided into 10 equidistant small subintervalls, the values of $f^{(k)}(x)$ at the nodes of these small subintervals are checked if they are sufficiently small against the maximal value of $f^{(k)}$ in order to reduce eventually the size of the reconstruction support. This test is given for the right boundary as (John et al., 2007):

$$
\left(\sum_{j=1}^{10}\left[\left(f^{(k)}\left(x_{n j}\right)\right)^{2}\right]+\left(f^{(k)}\left(x_{n+1}^{(k)}\right)\right)^{2}\right)^{1 / 2} \leq t_{\text {ol }} l_{\text {red }} f_{\text {max }}^{(k)}
$$

If equation A.11 is satisfied, the reconstruction support is reduced by setting $x_{n+1}^{(k+1)}=\left(x_{n}^{(k)}+x_{n+1}^{(k)}\right) / 2$,

otherwise the boundary remains unchanged. For the new interval $\left[x_{1}^{(k+1)}, x_{n+1}^{(k+1)}\right]$, the nodes are redistributed equidistantly.

It is further checked that any value of $f^{(k)}(x)$ at the nodes $x_{i}(i=1, \ldots, n+1)$ and at the midpoints of the subintervals are greater than a given tolerance $t_{\text {neg }}$ (equation A.12). This test is implemented to ensure the positivity of the PDFs.

$$
f_{\min , \max }:=\min _{j=1, \ldots, k} f_{\max }^{(j)}, \quad \frac{f^{(j)}\left(x_{i}\right)}{f_{\min , \max }} \geq \text { tol }_{n e g}, \quad \frac{f^{(j)}\left(x_{i 6}\right)}{f_{\min , \max }} \geq t_{\text {mol }}
$$

with $t_{\text {neg }} \leq 0$. The problem of the ill-conditioning of the linear system of equations is treated by regulizing the system. This is achieved by neglecting small singular values according to a given tolerance $t_{\text {tolng }}$ using a pseudo inverse routine of Matlab.

Note that in John et al. (2007), an equidistant grid is used for the distribution of the nodes $x_{i}$. This is not adapted for non-smooth and/or multimodal distributions. In the adaptive algorithm by (De Souza et al. 2010), this problem is solved by introducing a non-equidistant grid where the nodes are repositionned appropriately in order to capture accurately the PDF's critical domains.

This method is extensively described by John et al. (2007). The support of the target PDF $[a, b]$ is subdivided into $n$ subintervals such as $a=x_{1}<x_{2}<\cdots<x_{n+1}=b$. In each subinterval $\left[x_{i}, x_{i+1}\right]$, the target PDF is approximated by a piecewise polynomial $s^{(l)}(x)$ of degree $l$. The system of equations is detailed for cubic splines $(l=3)$. The unknowns are the four coefficients of the $n$ splines. A smooth transition at the boundaries of the interval is assumed meaning that the PDF, its first and second derivatives are null at the boundaries, this gives $2 \times 3$ equations. The continuity of the splines, their first and second derivatives at the nodes provides $3(n-1)$ equations. The remaining $(n-3)$ equations are supplemented by the moments. This leads to solve a $4 n \times 4 n$ ill-conditioned linear system.

Since the set of the known moments limits the number of splines, one has to compute the reconstruction in the optimal support of $f(x)$ thus, the reconstruction is computed iteratively starting from an initial reconstruction $f^{(0)}(x)$ in an initial interval $\left[x_{1}^{(0)}, x_{n+1}^{(0)}\right]$. 
In order to optimize the reconstruction support and thus increase the accuracy of the method, the values of the reconstructed PDF $f^{(k)}(x)$ are checked at the boundaries of the support $\left[x_{1}^{(k)}, x_{n+1}^{(k)}\right]$ (i.e. in the 405 subintervals $\left[x_{1}^{(k)}, x_{2}^{(k)}\right]$ and $\left.\left[x_{n}^{(k)}, x_{n+1}^{(k)}\right]\right)$. For this, these two subintervals are divided into 10 equidistant small subintervalls, the values of $f^{(k)}(x)$ at the nodes of these small subintervals are checked if they are sufficiently small against the maximal value of $f^{(k)}$ in order to reduce eventually the size of the reconstruction support. This test is given for the right boundary as (John et al., 2007):

$$
\left(\sum_{j=1}^{10}\left[\left(f^{(k)}\left(x_{n j}\right)\right)^{2}\right]+\left(f^{(k)}\left(x_{n+1}^{(k)}\right)\right)^{2}\right)^{1 / 2} \leq \operatorname{tol}_{\text {red }} f_{\text {max }}^{(k)}
$$

If equation A.13 is satisfied, the reconstruction support is reduced by setting $x_{n+1}^{(k+1)}=\left(x_{n}^{(k)}+x_{n+1}^{(k)}\right) / 2$,

otherwise the boundary remains unchanged. For the new interval $\left[x_{1}^{(k+1)}, x_{n+1}^{(k+1)}\right]$, the nodes are redistributed equidistantly.

It is further checked that any value of $f^{(k)}(x)$ at the nodes $x_{i}(i=1, \ldots, n+1)$ and at the midpoints of the subintervals are greater than a given tolerance $t_{\text {neg }}$ (equation A.14). This test is implemented to ensure the positivity of the PDFs.

$$
f_{\min , \max }:=\min _{j=1, \ldots, k} f_{\max }^{(j)}, \quad \frac{f^{(j)}\left(x_{i}\right)}{f_{\min , \max }} \geq \operatorname{tol}_{\text {neg }}, \quad \frac{f^{(j)}\left(x_{i 6}\right)}{f_{\min , \max }} \geq t o l_{\text {neg }}
$$

with $t_{\text {neg }} \leq 0$. The problem of the ill-conditioning of the linear system of equations is treated by regulizing the system. This is achieved by neglecting small singular values according to a given tolerance tol $_{\text {sing }}$ using a pseudo inverse routine of Matlab.

Note that in John et al. (2007), an equidistant grid is used for the distribution of the nodes $x_{i}$. This is not adapted for non-smooth and/or multimodal distributions. In the adaptive algorithm by (De Souza

et al., 2010), this problem is solved by introducing a non-equidistant grid where the nodes are repositionned appropriately in order to capture accurately the PDF's critical domains.

\section{Appendix B: Analytical solution of the PBE in the case of breakage process}

We give in this appendix the details for the analytical solution in its explicit form (equation 12.

By substituting equation 11 in 10 , we obtain :

$$
n(L, t)=\frac{1}{\sigma \sqrt{2 \pi}} \mathrm{e}^{-t L^{2}}\left(\mathrm{e}^{-\frac{1}{2}\left(\frac{L-\mu}{\sigma}\right)^{2}}+2 t \int_{L}^{\infty} L^{\prime} \mathrm{e}^{-\frac{1}{2}\left(\frac{L^{\prime}-\mu}{\sigma}\right)^{2}} d L^{\prime}\right)
$$

We note $I$ the integral term and proceed to the change of variable :

$$
L^{\prime}-\mu=\hat{L}^{\prime} \longmapsto L^{\prime}=\hat{L}^{\prime}+\mu \longmapsto d L^{\prime}=d \hat{L}^{\prime}
$$


The integral term is written as:

$$
I=\int \hat{L}^{\prime} e^{-\frac{1}{2 \sigma^{2}} \hat{L}^{\prime 2}} d \hat{L}^{\prime}+\mu \int e^{-\frac{1}{2 \sigma^{2}} \hat{L}^{\prime 2}} d \hat{L}^{\prime}
$$

Recall that :

$$
\int \mathrm{e}^{-a x^{2}} d x=\frac{\sqrt{\pi}}{2 \sqrt{a}} \operatorname{erf}(x \sqrt{a})
$$

and

$$
\int x \mathrm{e}^{-a x^{2}} d x=-\frac{1}{2 a} \mathrm{e}^{-a x^{2}}
$$

Thus :

$$
I=\sigma^{2} e^{-\frac{1}{2}\left(\frac{L-\mu}{\sigma}\right)^{2}}+\mu \sigma \sqrt{\frac{\pi}{2}}\left(1-\operatorname{erf}\left(\frac{1}{\sqrt{2}} \frac{L-\mu}{\sigma}\right)\right)
$$

By substituting equation B.6 in B.1, we obtain the final explicit solution given in equation 12 . 


\section{Nomenclature}

$\begin{array}{ll}a, b & \text { Lower and upper bounds of the reconstruction interval } \\ B_{n}, \tilde{B}_{n} & \text { Coefficients of the BKDF system of equations } \\ E(k) & \text { Global error function } \\ E_{r}(t) & \text { Mean absolute error for the reconstruction } \\ f(x) & \text { Probability Density Function } \\ f_{M}(x) & \text { The reconstructed Probability Density function } \\ g(x, \kappa, \lambda) & \text { Beta Probability Density Function } \\ h & \text { Bandwidth of the BKDF } \\ H[f] & \text { Shannon entropy } \\ I & \text { Number of BKDFs used for the reconstruction } \\ k, n & \text { moment's order } \\ K\left(x, x_{*}, h\right) & \text { Beta kernel density function } \\ L, L^{\prime} & \text { Polymer chains length } \\ L_{i} & \text { Abscissae of the Gaussian quadrature } \\ m & \text { Mean of the normal law } \\ M & \text { Number of nodes of the Gaussian quadrature } \\ n(L, t) & \text { Length based number density function } \\ N & \text { Length of the set of moments } \\ p_{i} & \text { Weight coefficient, } \in[0,1] \\ q & \text { Length of the discretized interval of reconstruction } \\ t, t_{i}, t_{f} & \text { Time, intial time, final time } \\ w_{i} & \text { Weights of the Gaussian quadrature } \\ x & \text { Variable of the PDF } \\ \bar{x} & \text { Centers of the BKDFs } \\ x_{*}, x_{i} & \end{array}$

\section{Greek Symbols}

$\alpha \quad$ Factor of proportionality for the breakage frequency

$\beta \quad$ Breakage kernel

$\gamma \quad$ Breakage frequency

$\Gamma \quad$ Gamma function

$\kappa, \lambda$ Parameters of the Beta Probability Density Function

$\mu \quad$ The set of moments

$\sigma \quad$ Standard deviation for the normal law

$\xi \quad$ Lagrange's multipliers 
BKDF Beta Kernel Density Function

CFD Computational Fluid Dynamics

CLD Chain Length Distribution

DP Degree of Polymerization

DQMOM Direct Quadrature Method of Moments

KDF Kernel Density Function

LQDMA Long Quotient-Modified Difference Algorithm

LSQLIN Constrained Linear Least Square

ME Maximum Entropy

MWD Molecular Weight Distribution

NNLS Non-Negative Least Square

PBE Population Balance Equation

PBM Polupation Balance Modelling

PD Product Difference Algorithm

PDF Probability Density Function

QMOM Quadrature Method of Moments

\section{References}

Abramov, R. (2006). A practical computational framework for the multidimensional moment-constrained maximum entropy principle. Journal of Computational Physics, 211, 198-209. URL: http://www. sciencedirect.com/science/article/pii/S0021999105002688, doi:10.1016/j.jcp.2005.05.008

Abramov, R. V. (2007). An improved algorithm for the multidimensional moment-constrained maximum 1] entropy problem. Journal of Computational Physics, 226, 621-644. URL: http://www.sciencedirect. com/science/article/pii/S0021999107001994.

Akhiezer, N. I. (1965). The Classical Moment Problem: N.I. Akhiezer. Oliver \& Boyd.

Athanassoulis, G. A., \& Gavriliadis, P. N. (2002). The truncated Hausdorff moment problem solved by 口 using kernel density functions. Probabilistic Engineering Mechanics, 17, 273-291. URL: http://www. sciencedirect.com/science/article/pii/S0266892002000127.

Biswas, P., \& Bhattacharya, A. K. (2010). Function reconstruction as a classical moment problem: a maximum entropy approach. Journal of Physics A: Mathematical and Theoretical, 43, 405003. URL: http://iopscience.iop.org/1751-8121/43/40/405003. doi:10.1088/1751-8113/43/40/405003

Bordás, R., John, V., Schmeyer, E., \& Thévenin, D. (2012). Numerical methods for the simulation of a coalescence-driven droplet size distribution. Theoretical and Computational Fluid Dynamics, 
घ 27, 253-271. URL: http://link.springer.com/article/10.1007/s00162-012-0275-9. doi 10.1007/ s00162-012-0275-9.

455 Bose, S. M., \& Git, Y. (2004). Mathematical modelling and computer simulation of linear polymer q degradation: Simple scissions. Macromolecular theory and simulations, 13, 453-473. URL: http: //onlinelibrary.wiley.com/doi/10.1002/mats.200300036/full.

De Souza, L. G. M., Janiga, G., John, V., \& Thévenin, D. (2010). Reconstruction of a distribution from a finite number of moments with an adaptive spline-based algorithm. Chemical Engineering Science, 65, 2741-2750. URL: http://www.sciencedirect.com/science/article/pii/S0009250910000163.

Dette, H. (1997). The Theory of Canonical Moments with Applications in Statistics, Probability, and Analysis. John Wiley \& Sons.

Diemer, R. B., \& Olson, J. H. (2002). A moment methodology for coagulation and breakage problems: Part 2moment models and distribution reconstruction. Chemical Engineering Science, 57, 2211-2228. URL: http://www.sciencedirect.com/science/article/pii/S0009250902001124.

Gavriliadis, P. N., \& Athanassoulis, G. A. (2003). Moment data can be analytically completed. Probabilis-

․ tic Engineering Mechanics, 18,329-338. URL:http://www.sciencedirect.com/science/article/pii/ S0266892003000468 doi:10.1016/j.probengmech.2003.07.001.

Gavriliadis, P. N., \& Athanassoulis, G. A. (2009). Moment information for probability distributions, without solving the moment problem, II: Main-mass, tails and shape approximation. Journal of computational and applied mathematics, 229, 7-15. URL: http://www.sciencedirect.com/science/article/ pii/S037704270800513X.

Gavriliadis, P. N., \& Athanassoulis, G. A. (2012). The truncated Stieltjes moment problem solved by using kernel density functions. Journal of Computational and Applied Mathematics, 236, 4193-4213. URL: http://www.sciencedirect.com/science/article/pii/S0377042712002257.

Gordon, R. G. (1968). Error Bounds in Equilibrium Statistical Mechanics. Journal of Mathematical

口 Physics, 9, 655-663. URL: http://scitation.aip.org/content/aip/journal/jmp/9/5/10.1063/1. 1664624, doi $10.1063 / 1.1664624$.

Hackbusch, W., John, V., Khachatryan, A., \& Suciu, C. (2012). A numerical method for the simulation of an aggregation-driven population balance system. International Journal for Numerical Methods in Fluids, 69,

1646-1660. URL: http://onlinelibrary.wiley.com/doi/10.1002/fld.2656/abstract, doi:10.1002/ fld.2656.

Hulburt, H. M., \& Katz, S. (1964). Some problems in particle technology: A statistical mechanical forq mulation. Chemical Engineering Science, 19, 555-574. URL: http://www.sciencedirect.com/science/ article/pii/0009250964850478. 
John, V., Angelov, I., Öncül, A. A., \& Thévenin, D. (2007). Techniques for the reconstruction of a distribution

ㅁ from a finite number of its moments. Chemical Engineering Science, 62, 2890-2904. URL: http://www. sciencedirect.com/science/article/pii/S0009250907002072.

John, V., \& Thein, F. (2012). On the efficiency and robustness of the core routine of the quadrature method

of moments (QMOM). Chemical Engineering Science, 75, 327-333. URL: http://www.sciencedirect. com/science/article/pii/S000925091200187X. doi 10.1016/j.ces.2012.03.024.

Kumar, S., \& Ramkrishna, D. (1996). On the solution of population balance equations by discretizationI. A

口 fixed pivot technique. Chemical Engineering Science, 51, 1311-1332. URL: http://www.sciencedirect. com/science/article/pii/0009250996884892, doi:10.1016/0009-2509(96)88489-2.

495 Lebaz, N., Cockx, A., Spérandio, M., \& Morchain, J. (2015). Population balance approach for the modelling of enzymatic hydrolysis of cellulose. The Canadian Journal of Chemical Engineering, 93, 276-284. URL: http://onlinelibrary.wiley.com/doi/10.1002/cjce.22088/abstract, doi 10.1002/cjce.22088.

Lee, K. W. (1983). Change of particle size distribution during Brownian coagulation. Journal of Col-

口 loid and Interface Science, 92, 315-325. URL: http://www.sciencedirect.com/science/article/pii/ 0021979783901534 .

Lin, Y., Lee, K., \& Matsoukas, T. (2002). Solution of the population balance equation using constant7 number Monte Carlo. Chemical Engineering Science, 57, 2241-2252. URL: http://www.sciencedirect. com/science/article/pii/S0009250902001148, doi 10.1016/S0009-2509(02)00114-8.

Madras, G., \& McCoy, B. J. (1998). Time evolution to similarity solutions for polymer degradation.

AIChE Journal, 44, 647-655. URL: http://onlinelibrary.wiley.com/doi/10.1002/aic.690440313/ abstract, doi:10.1002/aic.690440313.

Marchisio, D. L., \& Fox, R. O. (2005). Solution of population balance equations using the direct quadrature

q method of moments. Journal of Aerosol Science, 36, 43-73. URL: http://www.sciencedirect.com/ science/article/pii/S0021850204003052 //onlinelibrary.wiley.com/doi/10.1002/aic.690490517/abstract.

Marchisio, D. L., Vigil, R. D., \& Fox, R. O. (2003b). Quadrature method of moments for aggregationbreakage

口 processes. Journal of Colloid and Interface Science, 258, 322-334. URL: http://www.sciencedirect. 515 com/science/article/pii/S0021979702000541.

Massot, M., Laurent, F., Kah, D., \& De Chaisemartin, S. (2010). A robust moment method for evaluation of the disappearance rate of evaporating sprays. SIAM Journal on Applied Mathematics, 70, 3203-3234. URL: http://epubs.siam.org/doi/abs/10.1137/080740027 
McCoy, B. J., \& Madras, G. (2001). Discrete and continuous models for polymerization and depolymerization. Chemical Engineering Science, 56, 2831-2836. URL: http://www.sciencedirect.com/science/ article/pii/S0009250900005169, doi:10.1016/S0009-2509(00)00516-9.

McGraw, R. (1997). Description of Aerosol Dynamics by the Quadrature Method of Moments. Aerosol 口 Science and Technology, 27, 255-265. URL: http://dx.doi.org/10.1080/02786829708965471, doi:10. 1080/02786829708965471.

Mortier, S. T. F., De Beer, T., Gernaey, K. V., \& Nopens, I. (2014). Comparison of techniques for reconstruction of a distribution from moments in the context of a pharmaceutical drying process. Comput-

Tagliani, A. (1999). Hausdorff moment problem and maximum entropy: a unified approach. Applied Mathe口.

\section{S009630039810084X.}

Tagliani, A. (2001). Numerical aspects of finite Hausdorff moment problem by maximum entropy approach.

Applied mathematics and computation, 118, 133-149. URL: http://www.sciencedirect.com/science/ article/pii/S0096300399002106.

Wheeler, J. C. (1974). Modified moments and Gaussian quadratures. Rocky Mountain Journal of Mathematics, 4, 287-296. URL: http://projecteuclid.org/euclid.rmjm/1250130972.

Yuan, C., Laurent, F., \& Fox, R. O. (2012). An extended quadrature method of moments for population bal545 1. article/pii/S0021850212000699.

Ziff, R. M., \& McGrady, E. D. (1985). The kinetics of cluster fragmentation and depolymerisation. Journal 口 of Physics A: Mathematical and General, 18, 3027. URL: http://iopscience.iop.org/0305-4470/18/ $15 / 026$ 